\title{
Erratum to: Backbone chemical shift assignments of the acyl-acyl carrier protein intermediates of the fatty acid biosynthesis pathway of Plasmodium falciparum
}

Santosh Kumar Upadhyay • Ashish Misra •

Namita Surolia $\cdot$ Avadhesha Surolia .

Monica Sundd

Published online: 28 March 2010

(C) Springer Science+Business Media B.V. 2010

Erratum to: Biomol NMR Assign

DOI 10.1007/s12104-010-9212-2

Author would like to add acknowledgment as given below:

\section{Acknowledgment}

The work was supported by the NMR grant funded by the Department of Biotechnology, India.

The online version of the original article can be found under doi:10.1007/s12104-010-9212-2.

S. K. Upadhyay · A. Surolia · M. Sundd $(\bowtie)$

National Institute of Immunology, Aruna Asaf Ali Marg,

JNU Campus, New Delhi 110067, India

e-mail: monicasundd@nii.res.in

\section{A. Misra · A. Surolia}

Molecular Biophysics Unit, Indian Institute of Science,

Bangalore 560 012, India

N. Surolia

Molecular Biology and Genetics Unit, Jawaharlal Nehru Center

for Advanced Scientific Research, Bangalore 560 064, India 